\section{Reflections on the Yearbook from the 1990's to the Present}

\author{
M. J. Ball', J. S. Silva ${ }^{2}$ \\ 1 IBM Research, Baltimore, Maryland, USA \\ 2 Silva Consulting Services, Eldersburg, Maryland, USA
}

\section{Summary}

The authors highlight IMIA's progress over the past twenty years as a key bridging organization that translates health informatics theory into practice. In contrast, they describe that electronic health record (EHR) systems built in the 20th Century are not meeting the needs of clinical users. Moreover, these EHRs are not architected to keep pace with the rapid changes in the evolving health ecosystem. They conclude that 21st Century health IT systems need to be architected into an ecosystem-wide suite of interacting complex adaptive systems that support individuals, clinicians, managers and policy-makers with the high value/high usability computing paradigm that dominates the Internet today.

\section{Keywords}

Health ecosystem, EHRs, complex adaptive system, high value /

high usability paradigm, Internet of Things

Yearb Med Inform 2016;Suppl1:S10-1

http://dx.doi.org/10.15265/IYS-2016-s001

Published online May 20, 2016
In celebration of the IMIA yearbook, we have been invited to revisit two prefaces of the IMIA Yearbook that we authored during Dr. Ball's presidency of IMIA from 1992 to 1995. In the 1993 preface, we addressed the issue of broadening the IMIA membership by inviting several new types of members to IMIA to help support the activities of our organization and build bridges with other professionals in related areas of interest. The new categories included Universities, vendors, consultants, and professional organizations, such as WHO, IFIP, ISTACH, and IFMBE.

Special emphasis was placed by us on making IMIA a "Bridging Organization", encouraging inter-professional activity, and indeed working towards encouraging the concept of bringing informatics theory into practice. To this end, IMIA has worked diligently to engage and harness researchers within the international university research community, the World Health Organization and other entities mentioned. IMIA's outreach and engagement activities have continued to this day! We are delighted to report that the seeds we planted during these years, particularly during the General Assembly Meeting in Japan in 1993, bore valuable fruit. Under the inspiring leadership of IMIA's executive directors and presidents, much progress has been made.

Alexa Mc Cray and Jan Van Bemmel - the inaugural co-editors of the IMIA Yearbook - truly made a major contribution to our field with successful Yearbook publications. To this day, the IMIA Yearbook brings valuable material to our profession on an annual basis. The format and mode

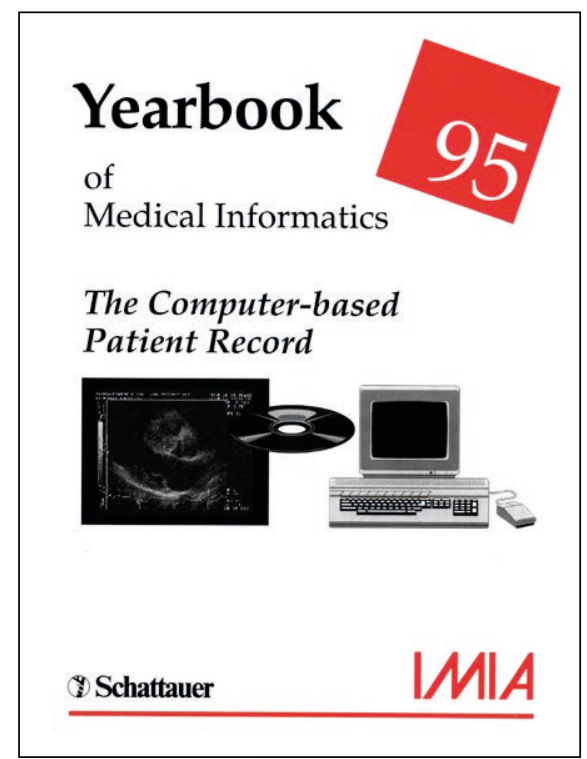

of distribution changed however to keep up with the demands of readers across the world and changing times.

In the 1995 preface, we discussed the major effort towards enabling computer-based patient records.

Much of what was discussed in that preface recognized the superb work that had already been done by Morrie Collen in these early years before 1995 . There was much enthusiasm within the community for rapid and universal implementation and acceptance of computer-based patient records and systems. Sadly to say, in concept, not much has changed in these past 20 years. The initial enthusiasm was rapidly replaced by skepticism and anger of clinicians, who found that these Electronic Health Record (EHR) systems did not provide useful information and actually made their work harder.

There has been substantial progress in some areas. The community is finally recognizing the clinicians' lament "EHRs don't think or work like I do" is accurate. Issues such as human factors, usability, interoperability, and change management have taken front and center stage. However, the changes at the bottom and top of health care systems are bringing new challenges to EHR systems. Today's EHRs have to address the role of the consumer/citizen and must support both patient-centric care (the bottom level where care decisions are 
actually made) and population health and predictive analytics (the top layer). Most EHRs were not architected to support this evolution; they were built on $20^{\text {th }}$ Century data technologies that were designed as isolated and vertically integrated stove pipes of health data.

In a recently contributed chapter to the fourth edition of Healthcare Information Management Systems entitled: Engineering the Next Generation of Health Systems, the authors strongly suggested that the health community has to understand itself in the context of a highly diverse health ecosystem. They identified three major changes that the community must embrace in the $21^{\text {st }}$ Century. In contrast to the current state of EHR systems, the authors highlight how the Internet is rapidly expanding to the "Internet of Things" (10 $10^{23}$ Bytes), how health care is migrating to the home, and how the high value for minimal user 'cost' (high value/high usability) is the dominant paradigm for the Web. The authors concluded that $21^{\text {st }}$ century health systems and their IT infrastructures must be re-architected into an ecosystem-wide set of interacting complex adaptive systems.

"One clear manifestation of the Web's adaptive behavior is the rapid emergence of cloud computing. These vast grids of always-on computing resources are fundamentally changing how companies purchase IT components and services. In many cases, fairly robust versions of software products are free, like Google Analytics, web conferencing systems, or the phone service Skype. As a result, today's users expect their "point of need" devices to access whatever information they need, wherever and whenever they need it, and conduct useful transactions with no learning curve; i.e., on Internet Time. For example, it is expected that a person can access his/her online banking services from his/her Smartphone or tablet, deposit checks, pay their bills and manage his/ her finances in a completely transparent way, all without taking a single training class or having to change his/her behavior. This high user value for minimal user cost (high value/ high usability) computing paradigm has enabled Smartphone and tablet computing to become the dominant model for user interactions with the Web. In fact, users expect these sorts of experience from their interactions with any IT. The health ecosystem must learn to play by these rules." [1] How do EHRs and health systems incorporate the plethora of available and emerging new tools, such as, IBM's Watson for health, voice recognition, analytics, decisions support, simulation for training, and cloud technologies? These new advances and mobile technologies could have a profound impact on all aspects of health care, particularly for clinicians and consumers at the points where they might make healthier decisions.

We close this preface with a question and a $21^{\text {st }}$ Century challenge to the IMIA community:

How do we build out and support a useful and usable health ecosystem that evolves in an affordable and sustainable fashion to meet the needs of all its constituents and ensure both patient safety and integrity?

\section{References}

1. Silva, JS and Ball, MJ, Chapter title" Engineering the Next Generation of Health Systems" in Healthcare Information Management Systems, 4th Ed. London,: Springer-Verlag ,pp 461-476, October 2015

Correspondence to:

Marion J. Ball Ed.D

Senior Advisor

Research Industry Specialist

Healthcare Informatics

IBM Research

Professor Emerita, Johns Hopkins University

5706 Coley Court

Baltimore, Maryland 21210, USA

Tel: + 14104337110

Fax: + 14104336314

Cell: + 14438025490

E-mail: marionball@us.ibm.com 